\title{
Behavioral Complexity Quantification (Becom-Q)
}

\author{
Dionisio de Niz, Min-Young Nam, and Julien Delange \\ SEI - Carnegie Mellon University \\ 4500 Fifth Avenue \\ Pittsburgh, PA, U.S.A. \\ dionisio@sei.cmu.edu,mnam@sei.cmu.edu, jdelange@sei.cmu.edu
}

\begin{abstract}
Todays' embedded and cyber-physical systems (CPS) rely heavily on complex software functions. While part of this complexity is unavoidable and caused by a growing number of functions (intrinsic complexity), another part is related to inappropriate design and development methods (called avoidable complexity). Indeed the early removal of avoidable complexity is one of the key challenges of software-intensive systems.

Over the years, several methods have been proposed to identify and quantify software complexity. However, current complexity measures rely mostly on the analysis of software structures and are not good predictors of software complexity and potential design flaws. This motivates the needs of new metrics of software complexity.

In this paper, we describe a new complexity metric based on the analysis of behavioral aspects of software we call Behavioral Analysis Quantification or Becom-Q for short. Specifically, we use advances in model-checking and model counting to quantify all possible behaviors that a software function may exhibit and use this quantification to model software complexity.
\end{abstract}

\section{INTRODUCTION}

The flexibility of software has created an explosion of productivity in terms of the amount of features that is possible to add to a system in an interval of time. This productivity is not only present in pure information systems but is also obtained in embedded and Cyber-Physical Systems (CPS) developed in the automotive, aerospace or avionics industries. For example, Volvo claims that $90 \%$ of their innovations happens in software [3]. Unfortunately, this flexibility creates also big opportunities for errors leading to an industry spending between $50 \%$ and $75 \%$ of the budget of a project in debugging, testing, and verification activities [15]. One of the key factors leading to this situation is the complexity of software. This complexity is separated in two main groups: the intrinsic complexity, related to the grow-

Permission to make digital or hard copies of all or part of this work for personal or classroom use is granted without fee provided that copies are not made or distributed for profit or commercial advantage and that copies bear this notice and the full citation on the first page. Copyrights for components of this work owned by others than ACM must be honored. Abstracting with credit is permitted. To copy otherwise, or republish, to post on servers or to redistribute to lists, requires prior specific permission and/or a fee. Request permissions from permissions@ acm.org.

WETSoM'16, May 16 2016, Austin, TX, USA

(c) 2016 ACM. ISBN 978-1-4503-4177-6/16/05 . \$ $\$ 15.00$

DOI: http://dx.doi.org/10.1145/2897695.2897700 ing number of functions and the avoidable complexity that is the result of inadequate design and development methods. While the former is necessary, the latter must be eliminated if we want to keep a sustainable innovation pace in CPS. Complex software is more difficult to understand, modify, and verify than simpler one. As a result, identifying, measuring, and managing software complexity is one of the great challenges in software engineering.

Unfortunately, current techniques to measure software complexity are mostly informal and requires human judgment. This is the case of complexity metrics based on software structure that capture elements such as coupling and cohesion of modules as well as fan-in and fan-out of the control and data flows. While these techniques are useful to guide design, they fail to capture the behavioral factors required to understand the algorithmic connection between modules and the complexity of module internals. In this paper we present a new approach to calculate software complexity that captures software behavioral characteristics. This approach takes advantage of advances in model checking and its use for model counting and quantitative information flow to quantify both the algorithmic complexity of module implementations and inter-module relationships. This approach not only eliminates human judgment but also can be completely automated providing a faithful quantification of the behavioral characteristic as an expression of all possible executions of the final implementation down to the $\mathrm{C}$ code. We call this approach Behavioral Complexity Quantification or Becom-Q for short.

Precise and automatic calculation of software complexity allow us to compare different versions of design artifacts in order to discover complexity that can be prevented that we call avoidable complexity. This complexity is not only present in program implementations but also in other software design artifacts such as state machines and architectural models used in Model-Based Engineering approaches. In this paper we first present the fundamentals of the complexity measuring technique, then we discuss how it is applied to program implementations in $\mathrm{C}$ and how to use it in design artifacts of high-level of abstraction.

\section{SHORTCOMINGS OF TRADITIONAL SW COMPLEXITY METRICS}

Current measures of software complexity are either based on the structure of the software or its size but neglects its behavior. In particular, Fenton and Neil [5] conducted statistical experiments on software projects that showed that the module size is not a good predictor of failures. Similarly, the 
authors of this paper showed that structural notions of complexity such as cyclomatic complexity [11] fails to identify failure-prone modules. However, in the same study they discovered some evidence to support that metrics such as SigFF have some correlation with the module failures. SigFF captures the number of new or modified signals (similar to messages or method calls) in a module [13]. Perhaps one of the most intriguing result from Fenton and Neil's work [5] is that the modules with the higher incidence of fault in prerelease testing are not the ones with the higher number of operational faults. Moreover, in their experiments the authors were able to identify a small number of rogue modules that were the most error-prone. These are the modules that seem to have some form of intrinsic complexity that metrics such as the cyclomatic complexity were not able to capture.

An alternative complexity point of view is offered by cognitive complexity $[16,9]$ that attempts to capture the complexity of the human process involved in the manipulation of software. Unfortunately current approaches in this area, such as assigning cognitive weights [16] to program structures, do not capture all the behavioral aspects of an algorithm. In particular, the complexity of the conditions in the branching structures as well as their relationship with the computation and other conditions is never captured. However, this new direction correctly highlights the character of software as information that needs to be processed. This character has been further explored by researchers that have followed an information-theoretic approach to software complexity $[2,6,8,7]$. This approach is also capable of assigning different weights to different information elements in the software. The theoretical underpinnings of this approach have a strong relationship with algorithmic complexity. In particular, it has been proven that the minimum expected length of a description of a string as described by ShannonFano encoding is an approximation of the Kolmogorov (more on this in Section 3) complexity of the string.

Unfortunately, the information theoretic techniques for software complexity are currently applied over an abstraction of the software that hides most of the targeted complexity. For instance, Allen et al. [2] apply this over hypergraphs of nodes, where the nodes represent sets of instruction sequences and the edges represent branching or modular communication. This means that the relationship between the computation and the branching conditions is hidden (which in the end captures key aspects of the behavior of software).

Understanding the behavior would provide a better and more accurate estimate of the complexity of a program. In this paper we present a new approach based on the entropybased approximation of the Kolmogorov complexity of the algorithm as implemented in a programming language. With this approximation we are able to measure the complexity of modules and combinations of modules. Our approach is independent on the programming language and can be applied to higher-level design artifacts such as state machines as soon as their operational semantics is completely specified. In particular, the tools we use are based on the $\mathrm{C}$ programming language, but we use translators from higher-level abstractions to transform it into $\mathrm{C}$ semantics (through code generators) in order to explore its behavior as translated into this language. In this paper we apply such an approach to software models captured using SCADE [1] that are then translated into $\mathrm{C}$ code. This allow us to have a precise early metrics to help us predict the quality of the final product down the road.

\section{KOLMOGOROV COMPLEXITY AND IN- FORMATION THEORY}

In this section we review the concepts of Kolmogorov complexity and information theory used to quantify program complexity. In particular, we first describe Kolmogorov complexity and then relate it to an information-entropy approximation.

In 1965, Kolmogorov created a definition of the intrinsic descriptive complexity of an object as the length of the shortest computer program that describes the object. This is known as the algorithmic complexity of an object. In practical terms, the object can be seen as a string and the program as some form of a formula or coding that can be used to reproduce the string. For instance, consider the following two sample strings from the book [4]:

1. 0101010101010101010101010101010101010101010101010101010101010101

2. 0110101000001001111001100110011111110011101111001100100100001000

In this case string 1 is only a repetition of the two digits 0132 times. In contrast, string 2, while seemingly random, is the initial part of the binary encoding of $\sqrt{2}-1^{1}$. In the extreme, a truly random string would need to be encoded as the string itself without any possibility of compressing its encoding.

Information theory allow us to capture an approximation on the complexity of a string through instantaneous compressing codes. A compressing code is an encoding of the alphabet of a language where the length of the encoding of a letter (or codeword) is inversely proportional to the relative frequency of the letter in messages in this language. For instance, the letters ' $\mathrm{E}$ ' and ' $\mathrm{Z}$ ' have a relative frequency of $12.702 \%$ and $0.074 \%$ respectively in the English language ${ }^{2}$. These encoding has the purpose of reducing the length of the encoding of texts in the language. For instance, for an alphabet of four letters A,B,C, and D, with a frequency of $\frac{1}{2}, \frac{1}{4}, \frac{1}{8}$, and $\frac{1}{8}$, a binary compressing encoding could be: $A \stackrel{1}{=} 0, B=10, C=110, D=111$. The average length (a.k.a. expected length) of a letter in a encoded text can be calculated by summing up the encoding length of each letter multiplied by its relative frequency. For this encoding the average size is: $\frac{1}{2} \times 1+\frac{1}{4} \times 2+\frac{1}{8} \times 3+\frac{1}{8} \times 3=1.75$ bits. This averaging equation is in fact the information entropy of the alphabet: $H(L)=-\sum_{l \in L} p(l) \log _{2}(p(l))$. This contrast with an average of 2 if all letters were encoded with a twobit codeword. This code is also an instantaneous code where no codeword is a prefix of any other codeword. This code allow us to compress a string such that the expected length (a.k.a. average length) of the letters of the compressed string is close to the entropy of the letters in the string, i.e.:

$$
E(\operatorname{Len}(x)) \geq H(x)
$$

where $H(x)$ can be interpreted as the uncertainty of knowing the next letter in a message (as predicted by their frequency in the language or how many bits, on average, I do not know about the next letter). $H(x)$ is in fact the minimum expected length of a codeword in the code. Hence, for a string

\footnotetext{
${ }^{1}$ It is easy to imagine the corresponding minimal programs for these two sequences.

${ }^{2}$ According to Wikipedia.
} 
of size $n$ the minimum expected length of a string encoded in this code is $n H(x)$. This is in fact an approximation of the Kolmogorov complexity $(\hat{K})$ of the string.

$$
\hat{K}(X)=n H(x)
$$

This allows us to evaluate the complexity of an algorithm based on the string of all possible outputs that it is able to generate. In other words, given the string of all possible outputs that an algorithm can generate, the complexity of this algorithm can be approximated as the expected length of the string encoded in an instantaneous code.

It is worth noting that, in practice, algorithms capture transformations from inputs to outputs. Taking this into account it is clear that the output string point of view must be modified to include the inputs. In the next section we introduce channel capacity as a way to do this.

\subsection{Channel Capacity}

The concept of channel capacity was created in information theory to capture the maximum amount of information that is possible to transmit through a noisy communication channel. A channel can be formalized [12] as a triplet $\left(I, O, C_{I, O}\right)$ where $I$ is the set of input values, $O$ is the set of output values, and $C_{I, O}$ is a $|I| \times|O|$ matrix known as the channel matrix where $C_{I, O}[i, o]=P(o \mid i)$ is the conditional probability of obtaining output $o$ given that the input is $i^{3}$.

This concept allows us to quantify the information that flows from input to outputs. This concept is also known as Quantitative Information Flow (QIF). QIF has been used to quantify the information leakage, if the input is considered a secret, and to quantify the influence that the input has over the outputs in an algorithm.

It is worth noting that this information flow captures how easy it is to predict the output based on the knowledge of the input. As a result, it also capture the complexity of the transformation of the inputs into outputs. Relating this back to the Kolmogorov complexity and instantaneous code, we can think of the string as a string of transformations $(i, o)$ instead of only a string of outputs. Then, instead of using the frequency of the letters in a instantaneous code we use the probabilities of the transformation $p(o \mid i)$ in the channel matrix. We will use an indirect technique to automatically calculate this taking advantage of recently developed tools for information leakage. To take advantage of this we first formalize the relationship between channel capacity and information leakage in the next section.

\subsection{Information Leakage}

Information leakage is a concept related to quantitative information flow where the input is considered a secret (we will use $s$ instead of $i$ to convey this) we want to preserve ${ }^{4}$. In this case, we want to investigate how likely it is to guess the secret (e.g. password) given that we are able to know the output of an (encryption) algorithm. In this case, if we obtain the entropy of both the secret (i.e., how difficult it is to guess the secret) $H(S)=-\sum_{s \in S} P(s) \log P(s)$ and the conditional uncertainty (entropy) of knowing the secret

\footnotetext{
${ }^{3}$ Each row sums up to one.

${ }^{4}$ The input can be divided into one part that is under the control of an attacker and another that is considered the secret.
}

given the output $H(S \mid O)=\sum_{o \in O} P(o) H(S \mid o)$, then it is possible to define leakage as

$$
\text { leakage }=H(S)-H(S \mid O)=I(S ; O)
$$

which captures the concept of leakage = initial uncertainty remaining uncertainty (after seeing output). This is equal to the mutual information between the secret and the output $(I(S ; O))$ as defined in information theory.

The symmetric nature of the mutual information allow us to also express 3 as:

$$
I(S ; O)=I(O ; S)=H(O)-H(O \mid S)
$$

which allows us to return back to the uncertainty of knowing the output given the input from the channel capacity. Note as well that 4 reduces the entropy of the output based on the input. This captures the "compression" notion of the instantaneous codes. Hence, the more mutual information (or leakage) the less compression was possible and the larger the Kolmogorov bound that maps back to the complexity of the algorithm.

When the probability distribution for $S$ is unknown we can assume the wost-case distribution which is the uniform distribution.

It is worth noting that for deterministic channels the value of $H(S \mid O)=0$ and hence leakage is reduced to $H(S)$. In reality, a number of algorithms generate different outputs for the same input (appearing non-deterministic) if the state of the system is different. We discuss how this is accounted for in the next section.

\subsection{Accounting for Program State}

In order to take into account the state of a program in its execution, it is necessary to execute the program multiple times with a sequence of inputs. This approach was taken by Marculescu et al. [10]. Specifically, in this paper the authors build a Markov model using the history of inputs as a state from which the next input is taken to model circuits. The authors noticed that different circuits required different lengths of history to be able to model a stable Markov model. The authors measured the entropy of the model and the entropy of adding another step in the history. To take into account the history, the step entropy was modeled as the entropy of a vector (of inputs) and the probability of the vectors was taken into account. The convergence of their step entropy was the indication that the model has converged as well. The length of the history define the order of the Markov model in what is called an lag- $k$ Markov chain.

To incorporate the internal state concept in the instantaneous coding approach to bounding the Kolmogorov complexity we can execute the program multiple times to create the output string. When we run the program numerous times, it would mean as if we are exploring the same behavior of the history in [10].

\section{AUTOMATING COMPLEXITY MEASURE- MENT}

In the previous section we established the relationship between information flow, output coding and Kolmogorov complexity. In this section we present our method to mea- 
sure an approximation of the Kolmogorov complexity using qifc [14], a tool used to measure information leakage.

Qifc is a tool developed to measure the quantitative information flow of a $\mathrm{C}$ program which in turn captures the information leakage of the program. We extended the method presented in [14] to quantify complexity based on its relationship with information leakage and channel capacity. In [14], the authors used a symbolic approach based on the Satisfiability Modulo Theories (SMT) to check the satisfiability of a logical formula that represents a $\mathrm{C}$ program. This approach is then extended into an \#SMT (or model counting based on SMT) by exploring assignment of values to the output on a bit-by-bit basis creating a tree of values that can be quickly explored. The implementation for $\mathrm{C}$ programs uses CBMC in order to unroll the loops in a bounded fashion.

We use qifc [14] to explore and obtain the QIF of C functions that are called multiple times to evaluate their internal states (stored in global or static variables).

\subsection{Number of Executions and Output Scope}

Determining the number of executions necessary to discover all output variations given the internal state of a program is a non-trivial issue. On the one hand, if we know that for each different internal state we could ensure that we will see different outputs, it would be possible to keep executing until we cannot observe new values. However, on the other hand, it is possible for a program to implement thresholds on states, say a counter reaching a threshold, where the program issues the same output for different counter values until it reaches the threshold. Then at this point it could generate a different output.

We identified three strategies to determine the number of executions needed to explore all behaviors and outputs of a program: output convergence, state exposure, and explicit state depth determination.

\subsubsection{Output Convergence}

Output convergence is the simplest of the strategies. This strategy simply keeps increasing the number of executions of a function until the number of output values converges. As discussed before, this strategy is blind to state thresholds.

\subsubsection{State Exposure}

This strategy involves modifying the program slightly to include the state variables (which values are preserved across executions) as explicit outputs. This has the advantage of a completely observable state for which we can detect all possible variations. This strategy has the disadvantage of being intrusive in the sense that it is necessary to modify the program to expose the output. In some development environment, such as code generated automatically from a model (as is the case of SCADE used in our experiments) state variables are accessible from the output sometimes in the form of an output pointer. This simplifies the task of taking them into account as regular output.

\subsubsection{State Depth Determination}

The state depth determination is a variation of the state exposure strategy but eliminating the modifications of the program. Specifically, in this case, a human interpretation of the program is required to determine how many repetitive executions is necessary to be able to explore all possible

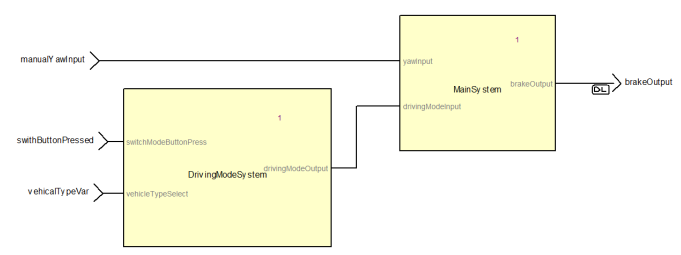

Figure 1: Top level SCADE model of ESC

states. This determination is easier to perform when the code is the product of a higher-level model such as a state machine.

\subsection{Complexity of Higher-Level Models}

We also used our complexity measuring method on highlevel models designed with SCADE [1]. In order to do this we used the code generation capabilities of the tool to translate the high-level model into $\mathrm{C}$ code and apply the complexity tools on the generated $\mathrm{C}$ code.

\section{ILLUSTRATIVE EXAMPLES}

In this section we present a number of higher-level model examples to which we apply our complexity measuring method. These examples are used to illustrate the issues of different complexity measurements of state encoding and repeated execution to explore all the states.

\subsection{Electronic Stability Control}

\subsubsection{Description}

The Electronic Stability Control (ESC) is a software component that computes the brake output value based on the yaw input from a sensor that represents dangerous driving dynamics such as under and over steering. Depending on the current choice of driving mode for a vehicle (sport, eco, offroad) the ESC module can behave differently. In particular, a sport car offers a driving mode where the oversteering is not corrected. This gives the driver the possibility of drifting the car and executing his own oversteering corrections. In addition, some vehicle may not have all the possible driving modes: a truck could have only a single OFFROAD driving mode.

\subsubsection{Architecture}

Figure 1 shows the top level model of the ESC. It is composed of three inputs, 1. manualYawInput: the input from the yaw sensor 2. switchButtonPressed: switch driving mode (offroad, comfort, eco, sport) 3. vehicalTypeVar: type of the vehicle executing the system (sedan, luxury, sport, truck)

It has one output value (brakeOutput) which represents the braking value. There are two subsystems: 1 . DrivingModeSystem, shown in Figure 2, gives the current driving mode value to the MainSystem. Figure 2 shows how the mode can be switched for each vehicle type within the set of driving modes that are available to each vehicle type. Such a software is a generic software that can be used across multiple types of vehicles. 2. MainSystem (Figure 3) uses the yaw input and the driving mode to decide on the current brake output. Whenever, the brake output changes, it also needs to be released before it can be ready to give a different type of brake output. The MainSystem is also a generic software and needs to handle all possible inputs into consideration. 


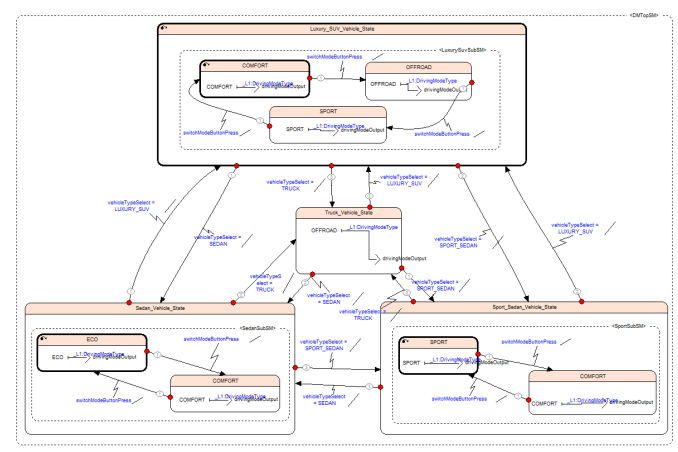

Figure 2: Driving Mode System of ESC

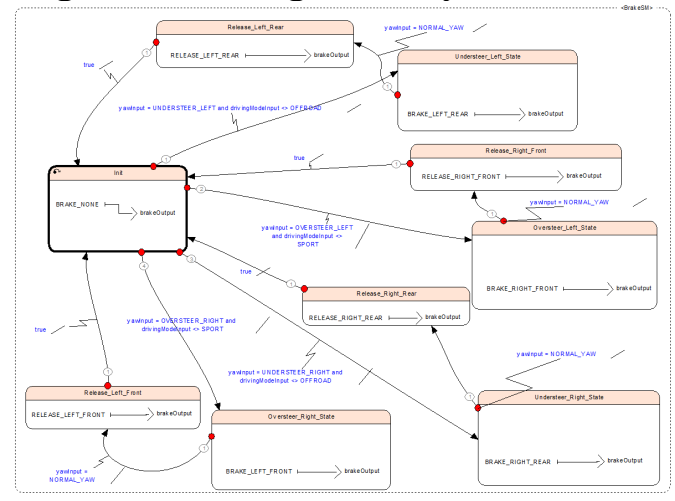

Figure 3: Main System of ESC

Table 1 shows all the values that are used in the ESC system.

\subsection{Train Door Example}

\subsubsection{Description}

The train door example is a door control system to safely open or close a door: the door cannot close if somebody is standing or stuck in front of the door and the system has safety features to avoid the user from being physically harmed.

The overall architecture is divided into two main components (controller and door) and a button that lets the user open or close the door. There is also a sensor that detects if somebody is standing in front of the door. It sends a signal to the controller to notify if somebody is present. If

\begin{tabular}{|c|c|}
\hline EnumType & Values \\
\hline & BRAKE_LEFT_FRONT \\
& BRAKE_LEFT_REAR \\
& BRAKE_RIGHT_FRONT \\
BrakeOut & BRAKE_RIGHT_REAR \\
& BRAKE_NONE \\
& RELEASE_LEFT_FRONT \\
& RELEASE_LEFT_REAR \\
& RELEASE_RIGHT_FRONT \\
& RELEASE_RIGHT_REAR \\
\hline \multirow{5}{*}{ DriveMode } & SPORT \\
& ECCO \\
& OFFROAD \\
Vehicle & COMFORT \\
& SEDAN \\
& LUXURY_SUV \\
& SPORT_SEDAN \\
YawMode & TRUCK \\
& UNDERSTEER_LEFT \\
& OVERSTEER_LEFT \\
& UNDERSTEER_RIGHT \\
& OVERSTEER_RIGHT \\
& NORMAL_YAW \\
\hline
\end{tabular}

Table 1: Enumeration Types and Values of ESC

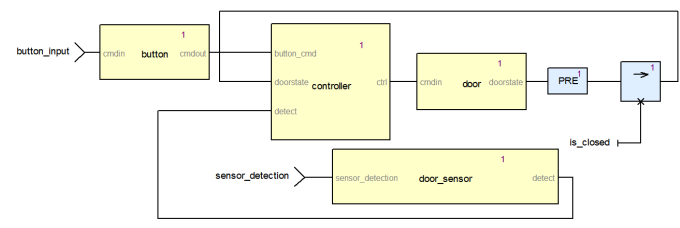

Figure 4: Top level SCADE model of Train Door

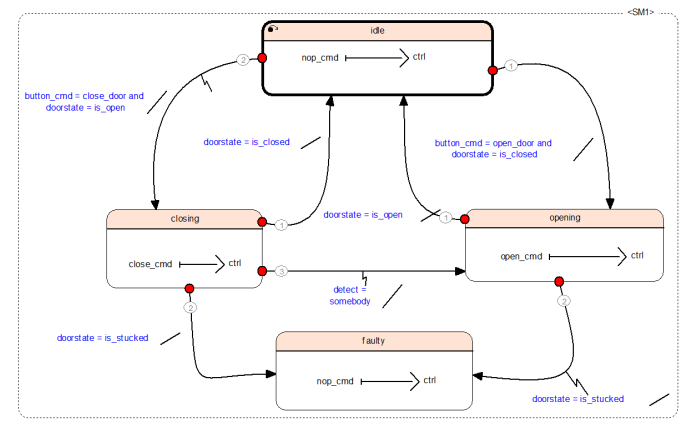

Figure 5: Controller System of Train Door

the door is closing when someone is detected, the door will change to opening.

\subsubsection{Architecture}

The controller sends commands to control the door (output named ctrl in Figure 5). The door has a motor that receives the commands from the controller. It also informs its current door state back to the controller through the output named doorstate in Figure 6.

The SCADE top level system model is shown in Figure 4 while the subsystems are shown in Figure 5 (controller) and Figure 6 (door) subsystems. The door model has a timer in the opening and closing state to simulate the time it takes to operate. When the timer reaches the door_timeout value, which we configured as 5 steps, it is assumed that the door has finished opening or closing.

On Figure 4, we can see the communication between modules. The button sends open_door or close_door value to the controller. The communication link between the controller and door can have the values of open_cmd, close_cmd, and nop_cmd. The controller checks both the state of the door and the input from the button to decide what control value to send: if somebody is standing in front of the door, the door will either stay open or will stop the closing process. The door sends its state back to the controller in one of the values of is_open, is_closed, is_moving, or is_stuck.

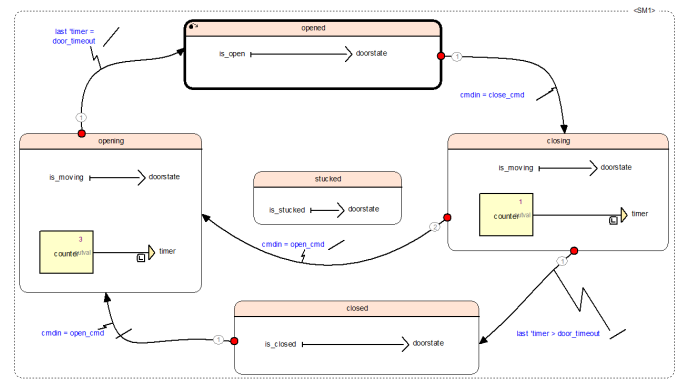

Figure 6: Door System of Train Door 


\begin{tabular}{|c|c|c|c|c|c|c|c|c|}
\hline$\#$ ex $\quad$ var & $\begin{array}{l}\text { contr. } \\
\text { state }\end{array}$ & \multicolumn{2}{|c|}{$\begin{array}{l}\text { contr. } \\
\text { output }\end{array}$} & \multicolumn{2}{|c|}{$\begin{array}{l}\text { door } \\
\text { state }\end{array}$} & \multicolumn{2}{|c|}{$\begin{array}{l}\text { door } \\
\text { output }\end{array}$} & total \\
\hline $\begin{array}{l}\text { Single } \\
\text { Multipl }\end{array}$ & 11 & \multirow{2}{*}{\multicolumn{2}{|c|}{$\begin{array}{c}1 \\
1.585\end{array}$}} & & & \multirow{2}{*}{\multicolumn{2}{|c|}{$\begin{array}{c}0 \\
1 \\
1.585\end{array}$}} & \multirow{2}{*}{$\begin{array}{c}2 \\
5.755 \\
6.755\end{array}$} \\
\hline $\begin{array}{l}\text { Multiple (7) } \\
\text { Multiple (8) }\end{array}$ & $\begin{array}{l}1.585 \\
1.585\end{array}$ & & & & & & & \\
\hline \multicolumn{9}{|c|}{ Table 2: Train Door Model QFF (bits) } \\
\hline ex var & $\begin{array}{l}\text { brake } \\
\text { butput }\end{array}$ & $\begin{array}{l}\text { car } \\
\text { type }\end{array}$ & $\begin{array}{c}\mathrm{si} \\
\mathrm{mc}\end{array}$ & & & & \begin{tabular}{|l} 
spor \\
mod
\end{tabular} & total \\
\hline ngle & 2.32 & 0 & & & & & 1 & $\begin{array}{l}7.32 \\
8775\end{array}$ \\
\hline
\end{tabular}

Table 3: ESC QIF (bits)

\subsection{Complexity Measurement Demonstrations}

\subsubsection{Execution Repetitions}

In this demonstration we show the effect of the number of execution on the QIF of our models: the train door (section 5.2) and the Electronic Stability Control (section 5.1).

Table 2 shows the effect of multiple executions in the door model. In particular it shows that it is necessary to execute the model multiple times to make sure that we get the maximum QIF. In this case we applied the state exposure strategy including state variables as part of the observable output. In particular, the observable output includes two output variables and two (exposed) state variables. Once the internal state is exposed we apply the convergence strategy to find the maximum number of execution necessary to reach the maximum QIF. The convergence strategy can also be combined with the state depth determination strategy by checking the number of possible values for each variable and making sure that each value is reachable. Once we discover the number of iterations that is required for a complete analysis, that in this case is 10 , we then use this iteration number for other cases where we may get less QIF values.

For the ESC, we only needed to execute twice to get the maximum QIF value for the significant variables shown in Table 3. In this case, it was enough to use the state depth determination strategy to reason about the number of execution necessary to obtain the maximum QIF. The determination was verified in the experiment.

\subsubsection{Measuring Complexity of Module Dependen- cies}

In this experiment we measure the complexity effect of using modules connected together. This helps us understand the effect of module relationships and structural complexity that includes the algorithmic complexity as well. In particular, we use the ESC example to explore the complexity of using a generic model for cases that use only part of its functionality.

Table 4 shows the complexity of the generic software by checking the QIF values for the same generic software but with different input constraints that represent the driving modes for the different vehicles in the ESC model . The vehicalTypeVar input (Figure 1) is now constant when checked for each type of vehicle. Decreased values in QIF means that

\begin{tabular}{|l|c|c|c|c|c|c|}
\hline constr. & $\begin{array}{c}\text { brake } \\
\text { output }\end{array}$ & $\begin{array}{c}\text { car } \\
\text { type }\end{array}$ & $\begin{array}{c}\text { suv } \\
\text { mode }\end{array}$ & $\begin{array}{c}\text { sedan } \\
\text { mode }\end{array}$ & $\begin{array}{c}\text { sports } \\
\text { mode }\end{array}$ & total \\
\hline Generic & 3.170 & 2 & 1.585 & 1 & 1 & 8.755 \\
SUV & 3.170 & 0 & 1.585 & 0 & 0 & 4.755 \\
Sedan & 3.170 & 0 & 0 & 1 & 0 & 4.170 \\
Sports & 3.170 & 0 & 0 & 0 & 1 & 4.170 \\
Truck & 2.322 & 0 & 0 & 0 & 0 & 2.322 \\
\hline
\end{tabular}

Table 4: ESC QIF (bits) with input constraint

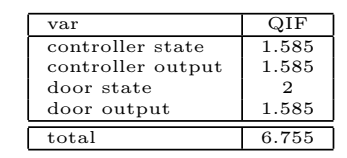

Table 5: Door QIF (bits) - Enumerators

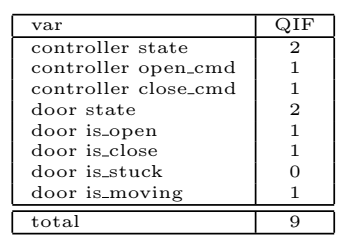

Table 6: Door QIF (bits) - Booleans not all states were reachable in the generic software. The QIF difference represents the unnecessary software complexity cost that is paid for using a generic software instead of developing a specialized module for each vehicle model.

\subsection{Structural Correctness Complexity Quan- tification}

\subsubsection{Measuring the Complexity of State Represen- tations}

In this experiment we measure the complexity of different state representations in the same model. The objective of this experiment is to capture common design alternative and the complexity of these alternatives.

Table 5 and Table 6 shows a comparison of how complexity increases when comparing the original train door model with an alternative implementation that uses booleans instead of enumerations to represent the state and commands of the door. This can be measured by the increase in the total QIF of significant variables.

As a first step, we are considering two implementations for each language: 1. One with commands specified using well-defined data types. For example, each communication will be defined using an enumeration type. 2. One with commands specified using boolean variables. In that case, each potential value for the command will require at least two boolean values.

To illustrate the variation, let us consider the door_cmd communication. When implementing using a well-defined data type, the data type will be an enumeration where the value can be open or close. The communication will then transport one value of this type. On the other hand, when implementing with boolean variables, we do not define any data type but communicate with (at least) two boolean variables (open, close) which will be set to true or false.

Then, the goal of this exercise will be to see the support of enumeration for each tool, evaluate the underlying validation or verification support and also investigate the potential support for generating tests.

As the second variation (using boolean variables) should be more complex, toolsuites could provide appropriate features to generate tests. Such an example would then show that the test vector for the boolean-values only is bigger than when using well-defined data types.

\subsubsection{Complexity Tradeoffs}

In this last experiment we evaluate the difference of using a generic model for the ESC versus using specialized models for each vehicle type. In the generic model, the code for all cars is included while in a specialized model, only the code specific to a type of car is included. The objective is to show the cost of complexity for managing generic models: while it can be easier to manage (one single model to manage for a complete line of several products), it can be more complex to 


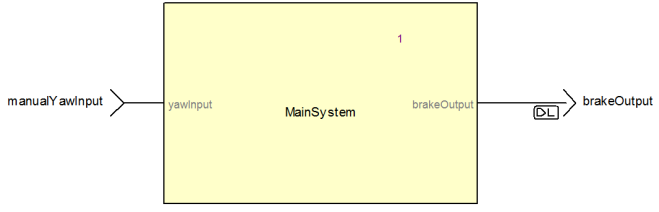

Figure 7: ESC SCADE Model - Truck only

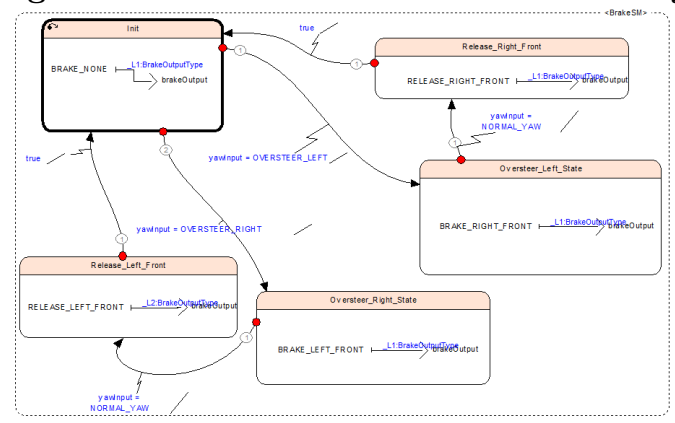

Figure 8: Main System of ESC - Truck only

maintain (e.g. a change for one type of car might introduce unexpected changes on other types).

We compare the QIF results of the generic model and two specialized ESC models: one for the truck car type, another for the SUV car type.

Figure 8 show the case for trucks. Since truck only allows for OFFROAD driving mode, it does not need a subsystem for selecting the driving mode. The top system is simplified accordingly and the MainSystem is also simplified to include only the possible brake output states for trucks.

The specialized model for SUV is shown in Figure 9 and Figure 10. In the DrivingModeSystem, only the state machine for the SUV remains. The MainSystem is the same as Figure 3 because every state is reachable for SUVs.

Although each of the customized models for truck and SUV is different from the generic model, the total QIF of significant variables remains the same when compared with the results shown in Table 4. In other words, the total QIF of the customized software is the same as when the generic software is used with matching constraints. This is because the significant variables that gave 0 QIF for the generic software are now removed in the customized software to reduce complexity. Still, the customized software is easier to understand due to fact that there are less significant variables. This is in fact, one aspect that our quantification method is currently not able to capture.

\section{CONCLUSION}

In this paper we presented a new method to quantify software complexity using advances in model-checking and model counting to take into account behavioral aspects of

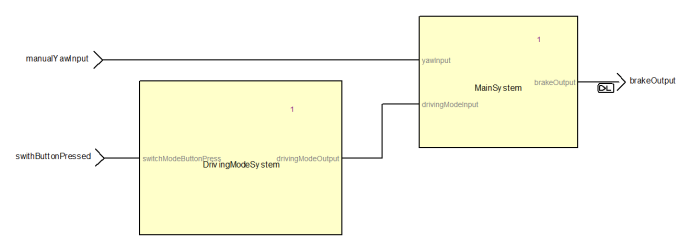

Figure 9: ESC SCADE Model - SUV only

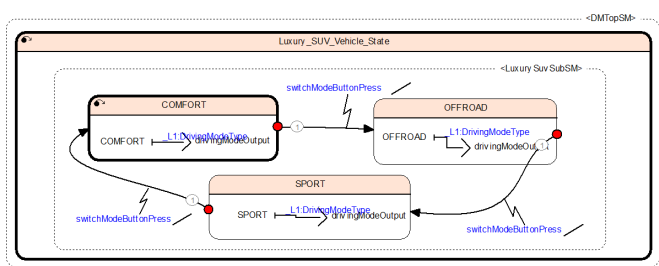

Figure 10: Driving Mode of ESC - SUV only

software. This method is designed to overcome the shortcomings of traditional complexity metrics that focus exclusively on software structures. In the paper we described the relationship of model counting and quantitative information flow techniques to information theory and the Kolmogorov complexity of algorithms and how this is used to quantify complexity. We then show how we use tools based on this techniques to quantify the complexity of software models developed at a higher level of abstraction with SCADE. This new method allows us to show how different design decisions such as choices to encode state in the system can lead to different levels of complexity and how our method is able to quantify the difference.

\section{ACKNOWLEDGMENTS}

Copyright 2016 ACM

This material is based upon work funded and supported by the Department of Defense under Contract No. FA872105-C-0003 with Carnegie Mellon University for the operation of the Software Engineering Institute, a federally funded research and development center. [Distribution Statement A] This material has been approved for public release and unlimited distribution. Please see Copyright notice for non-US Government use and distribution.

DM-0003378

\section{REFERENCES}

P. A. Abdulla, J. Deneux, G. Stålmarck, H. Agren, and O. Åkerlund. Designing safe, reliable systems using SCADE. In Leveraging Applications of
Formal Methods. Springer, 2006 .

[2] E. B. Allen, S. Gottipati, and R. Govindarajan. Measuring size, complexity, and coupling of hypergraph abstractions of software: An information-theory approach. Software Quality Journal, 15(2), 2007.

[3] J. Bosch. From opinions to facts: Building prodcuts customers actually use.

[4] T. M. Cover and J. A. Thomas. Elements of Information Theory. Wiley-Interscience, New York, NY, USA, 2006.

5] N. E. Fenton and M. Neil. Software metrics: successes, failures and new directions. Journal of Systems and Software, 47(23), 1999.

[6] Renato R. Gonzalez. A unified metric of software complexity: Measuring productivity, quality, and value. J. Syst. Softw., 29(1):17-37, April 1995.

7] Harrison. An entropy-based measure of software complexity. Software Engineering, IEEE Transactions on, 18(11), Nov 1992.

[8] K. Kim, Y. Shin, and C. Wu. Complexity measures for object-oriented program based on the entropy. In Software Engineering Conference, 1995. Proceedings., 1995 Asia Pacific, 1995.

[9] D. S. Kushwaha and A. K. Misra. Improved cognitive information complexity measure: a metric that establishes program comprehension complexity measure: a metric that establishes program co
effort. ACM SIGSOFT Software Engineering Notes, 31(5), 2006.

[10] R. Marculescu, D. Marculescu, and M. Pedram. Composite sequence compaction for finite-state machines using block entropy and high-order markov models. In International Symposium on Low Power Electronics and Design, markov.

[11] T. J. McCabe. A complexity measure. IEEE Trans. Softw. Eng., 2(4):308-320, July 1976.

[12] Z. Meng and G. Smith. Calculating bounds on information leakage using two-bit patterns. In ACM SIGPLAN 6th Workshop on Programming Languages two-bit patterns. In $A C M$ SIGPL

[13] N. Ohlsson and H. Alberg. Predicting fault-prone software modules in N. Ohlsson and H. Alberg. Predicting fault-prone software
telephone switches. IEEE Trans. Softw. Eng., 22(12), 1996.

[14] Q.-S. Phan and P. Malacaria. Abstract model counting: A novel approach for quantification of information leaks. In ACM Symposium on Information, Computer and Communications Security, 2014.

[15] RTI. The economic impacts of inadequate infrastructure for software testing. Technical report, National Institute of Standards and Technology.

[16] J. Shao and Y. Wang. A new measure of software complexity based on cognitive weights. Electrical and Computer Engineering, Canadian Journal of, $28(2), 2003$. 